\title{
Evaluation of Automatically Identified Index Terms for Browsing Electronic Documents ${ }^{1}$
}

\author{
Nina Wacholder, Judith L. Klavans and David K. Evans \\ Columbia University \\ Department of Computer Science and \\ Center for Research on Information Access
}

\begin{abstract}
1. Abstract
We present an evaluation of domainindependent natural language tools for use in the identification of significant concepts in documents. Using qualitative evaluation, we compare three shallow processing methods for extracting index terms, i.e., terms that can be used to model the content of documents. We focus on two criteria: quality and coverage. In terms of quality alone, our results show that technical term (TT) extraction [Justeson and Katz 1995] receives the highest rating. However, in terms of a combined quality and coverage metric, the Head Sorting (HS) method, described in [Wacholder 1998], outperforms both other methods, keyword (KW) and TT.
\end{abstract}

\section{Introduction}

In this paper, we consider the problem of how to evaluate the automatic identification of index terms that have been derived without recourse to lexicons or to other kinds of domain-specific information. By index terms, we mean natural language expressions that constitute a meaningful representation of a document for humans. The premise of this research is that if significant topics coherently represent information in a document, these topics can be used as index terms that approximate the content of individual documents in large collections of electronic documents.

We compare three shallow processing methods for identifying index terms:

- Keywords (KW) are terms identified by counting frequency of stemmed words in a document;
- Technical terms (TT) are noun phrases (NPs) or subparts of NPs repeated more than twice in a document [Justeson and Katz 1995];

- Head sorted terms (HS) are identified by a method in which simplex noun phrases (as defined below) are sorted by head and then ranked in decreasing order of frequency [Wacholder 1998].

The three methods that we evaluated are domain-independent in that they use statistical and/or linguistic properties that apply to any natural language document in any field. These methods are also corpus-independent, in that the ranking of terms for an individual document is not dependent on properties of the corpus.

\subsection{Overview of methods and results}

Subjects were drawn from two groups: professionals and students. Professionals included librarians and publishing professionals familiar with both manual and automatic text indexing. Students included undergraduate and graduate students with a variety of academic interests.

To assess terms, we used a standard qualitative ranking technique. We presented subjects with an article and a list of terms identified by one of the three methods. Subjects were asked to answer the following general question: "Would this term be useful in an electronic index for this article?" Terms were rated on a scale of 1 to 5 , where 1 indicates a high quality term that should definitely be included in the index and 5 indicates a junk term that definitely should not be included. For ex-

\footnotetext{
${ }^{1}$ This research was partly funded by NSF IRI 97-12069, "Automatic identification of significant topics in domain independent full text documents" and NSF IRI 97-53054, "Computationally tractable methods for document analysis".
} 
ample, the phrase court-approved affirmative action plans received an average rating of 1 from the professionals, meaning that it was ranked as useful for the article; the $\mathrm{KW}$ affirmative received an average rating of 3.75 , meaning that it was less useful; and the $\mathrm{KW}$ action received an average ranking of 4.5 , meaning that it was not useful.

The goal of our research is to determine which method, or combination of methods, provides the best results. We measure results in terms of two criteria: quality and coverage. By quality, we mean that evaluators ranked terms high on the 1 to 5 scale from highest to lowest. By coverage, we mean the thoroughness with which the terms cover the significant topics in the document. Our methodology permits us to measure both criteria, as shown in Figure 4.

Our results from both the professionals and students show that TTs are superior with respect to quality; however, there are only a small number of TTs per document, so they do not provide adequate coverage in that they are not fully representative of the document as a whole. In contrast, KWs provide good coverage but relatively poor quality in that $\mathrm{KWs}$ are vague, and not well filtered. SNPs, which have been sorted using HS and filtered, provide a better balance of quality and coverage.

From our study, we draw the following conclusions:

- The KW approach identifies some useful index terms, but they are mixed in with a large number of low-ranked terms.

- The TT approach identifies high quality terms, but with low coverage, i.e., relatively few indexing terms.

- The HS approach achieves a balance between quality and coverage.

\section{Domain-independent metrics for identi- fying significant topics}

In order to identify significant topics in a document, a significance measure is needed, i.e., a method for determining which concepts in the document are relatively important for a given task. The need to determine the importance of a particular concept within a document is motivated by a range of applications, including information retrieval [Salton 1989], automatic determination of authorship [Mosteller and Wallace 1963], similarity metrics for cross-document clustering [Hatzivassiloglou et al. 1999], automatic indexing [Hodges et al. 1996] and input to summarization [Paice 1990].

For example, one of the earlier applications using frequency for identifying significant topics in a document was proposed by [Luhn 1958] for use in creating automatic abstracts. For each document, a list of stoplisted stems was created, and ranked by frequency; the most frequent keywords were used to identify significant sentences in the original document. Luhn's premise was that emphasis, as indicated by repetition of words and collocation is an indicator of significance. Namely, "the more often certain words are found in each other's company within a sentence, the more significance may be attributed to each of these words." This basic observation, although refined extensively by later summarization techniques (as reviewed in [Paice 1990]), relies on the capability of identifying significant concepts.

The standard IR technique known as tf*idf [Salton 1989] seeks to identify documents relevant to a particular query by relativizing keyword frequency in a document as compared to frequency in a corpus. This method can be used to locate at least some important concepts in full text. Although it has been effective for information retrieval, for other applications, such as human-oriented indexing, this technique is impractical. Ambiguity of stems (trad might refer to trader or tradition) and of isolated words (state might be a political entity or a mode of being) means that lists of keywords have not usually been used to represent the content of a document to human beings. Furthermore, humans have a difficult time processing stems and parts of words out of phrasal context.

The technical term (TT) method, another technique for identification of significant terms in text that can be used as index terms was introduced by [Justeson and Katz 1995], who developed an algorithm for identifying repeated multi-word phrases such as central processing unit in the computer domain or word sense in the lexical semantic domain. 
This algorithm identifies candidate TTs in a corpus by locating NPs consisting of nouns, adjectives, and sometimes prepositional phrases. TTs are defined as those NPs, or their subparts, which occur above some frequency threshold in a corpus. However, as [Boguraev and Kennedy 1998] observe, the TT technique may not characterize the full content of documents. Indeed, even in a technical document, TTs do not provide adequate coverage of the NPs in a document that contribute to its content, especially since TTs are by definition multi-word. A truly domain-general method should apply to both technical and nontechnical documents. The relevant difference between technical and non-technical documents is that in technical documents, many of the topics which are significant to the document as a whole may be also TTs.

[Wacholder 1998] proposed the method of Head Sorting for identifying significant topics that can be used to represent a source document. HS also uses a frequency measure to provide an approximation of topic significance. However, instead of counting frequency of stems or repetition of word sequences, this method counts frequency of a relatively easily identified grammatical element, heads of simplex noun phrases (SNPs). For common NPs (NPs whose head is a common noun), an SNP is a maximal NP that includes premodifiers such as determiners and possessives but not post-nominal constituents such as prepositions or relativizers. For example, the well-known book is an SNP but the well-known book on asteroids includes two SNPs, wellknown book and asteroids. For proper names, an SNP is a name that refers to a single entity. For example, Museum of the City of New York, the name of an organization, is an SNP even though the organizational name incorporates a city name. Others, such as [Church 1988], have discussed a similar concept, sometimes called simple or base NPs.

The HS approach is based on the assumption that nominal elements can be used to convey the gist of a document. SNPs, which are semantically and syntactically coherent, appear to be at a good level of detail for content representation of the document. "
SNPs are identified by a system [Evans 1998; Evans et al. 2000] which sequentially parses text that has been tagged with part of speech using a finite state machine. Next, the complete list of SNPs identified in a document is sorted by the head of the phrase, which, at least for English-language common SNPs, is almost always the last word. The intuitive justification for sorting SNPs by head is based on the fundamental linguistic distinction between head and modifier: in general, a head makes a greater contribution to the syntax and semantics of a phrase than does a modifier. This linguistic insight can be extended to the document level. If, as a practical matter, it is necessary to rank the contribution to a whole document made by the sequence of words constituting an NP, the head should be ranked more highly than other words in the phrase. This distinction is important in linguistic theory; for example, [Jackendoff 1977] discusses the relationship of heads and modifiers in phrase structure. It is also important in NLP, where, for example, [Strzalkowski 1997] and [Evans and Zhai 1996] have used the distinction between heads and modifiers to add query terms to information retrieval systems.

Powerful corpus processing techniques have been developed to measure deviance from an average occurrence or co-occurrence in the corpus. In this paper we chose to evaluate methods that depend only on document-internal data, independent of corpus, domain or genre. We therefore did not use, for example, tf*idf, the purely statistical technique that is the used by most information retrieval systems, or [Smadja 1993], a hybrid statistical and symbolic technique for identifying collocations.

\section{Experimental Method}

To evaluate techniques, we performed a qualitative user evaluation in which the terms identified by each method were compared for usefulness as index terms.

\subsection{Subjects}

We performed our study with librarians, publishing professionals and undergraduate and graduate students at our university. 29 subjects participated in the study: 7 librarians and publishing professionals and 22 students. 


\subsection{Data}

For this experiment, we selected three articles from the 1990 Wall Street Journal contained in the Tipster collection of documents. The articles were about 500 words in length.

To compare methods, each article was processed three times: 1) with SMART to identify stemmed keywords [Salton 1989]; 2) with an implementation of the TT algorithm based on [Justeson and Katz 1995]; and 3) with our implementation of the HS method. Output for one article is shown in Appendix A. Figure 1 shows the articles selected, their length in words and the number of index terms from each method for each article presented to the subjects.

\begin{tabular}{|c|c|c|c|c|}
\hline DOC & words & KW & TT & HS \\
\hline $\mathbf{4 1 5 - 0 1 0 9}$ & 509 & 63 & 4 & 49 \\
\hline $\mathbf{5 1 6 - 0 0 4 3}$ & 594 & 51 & 9 & 54 \\
\hline $\mathbf{5 1 7 - 0 0 6 2}$ & 514 & 52 & 8 & 57 \\
\hline
\end{tabular}

Figure 1: Word and term count, by type, per article

The number of TTs is much lower than the number of KWs or HSs. This presented us with a problem: on the one hand, we were concerned about preserving the integrity of the three methods, each of which has their own logic, and at the same time, we were concerned to present lists that were balanced relative to each other. Toward this end, we made several decisions about presentation of the data:

1. Threshold: So that no bias would be unintentionally introduced, we presented subjects with all terms output by each method, up to a specified cut-off poinHowever, using lists of equal length for each method would have necessitated either omitting HSs and KWs or changing the definition of TTs. Therefore we made the following decisions:

- For TTs, we included all identified terms;

- For HSs, we included all terms whose head occurred more than once in the document;
- For KWs, we included all terms in order of decreasing frequency, up to the point where we observed diminishing quality and where the number of KWs approximated the number of HSs.

2. Order: For the $\mathrm{KW}$ and TT approach, order is not significant. However, for the HS approach, the grouping together of phrases with common heads is, we claim, one of the advantages of the method. We therefore alphabetized the KWs and TTs in standard left to right order and alphabetized the HSs by head, e.g., trust account precedes money market fund.

3. Morphological expansion: The KW approach identifies stems which represent a set of one or more morphological variants of the stem. Since in some cases the stem is not an English word, we expanded each stem to include the morphological variants that actually occurred in the article. For example, for the stem reject, we listed rejected and rejecting but did not list rejects, which did not occur in the article.

\subsection{Presentation to subjects}

Each subject was presented with three articles. For one article, the subject received a head sorted list of HSs; for another article, the subject received a list of technical terms, and for the third article, the subject saw a list of keywords. No time limit was placed on the task.

\section{Results}

Our results for the three types of terms, by document, are shown in Figure 2. Although we asked subjects to rate three articles, some volunteers rated only two. All results were included.

\begin{tabular}{|l|c|c|c|}
\hline Doc & $\begin{array}{c}\text { Avg } \\
\text { KW } \\
\text { rating }\end{array}$ & $\begin{array}{c}\text { Avg } \\
\text { TT } \\
\text { rating }\end{array}$ & $\begin{array}{c}\text { Avg } \\
\text { HS } \\
\text { rating }\end{array}$ \\
\hline $900405-0109$ & 3.08 & 1.45 & 2.71 \\
\hline $900516-0043$ & 3.73 & 2.19 & 2.71 \\
\hline $900517-0062$ & 2.98 & 1.7 & 3.25 \\
\hline Avg of Avgs & $\mathbf{3 . 2 7}$ & $\mathbf{1 . 7 9}$ & $\mathbf{2 . 8 9}$ \\
\hline
\end{tabular}

Figure 2: Average ratings of 3 types of index terms 


\subsection{Quality}

For the three lists of index terms, TTs received the highest ratings for all three documents-an average of 1.79 on the scale of 1 to 5 , with 1 being the best rating. HS came in second, with an average of 2.89 , and $\mathrm{KW}$ came in last with an average of 3.27 . It should be noted that averaging the average conceals the fact that the number of TTs is much lower than the other two types of terms, as shown in Figure 1.

Figure 3 (included before Appendix A) shows cumulative rankings of terms by method. The $\mathrm{X}$ axis represents ratings awarded by subjects. The $\mathrm{Y}$ axis reflects the percentage of terms receiving a given rank or better. All data series must reach $100 \%$ since every term has been assigned a rating by the evaluators. At any given data point, a larger value indicates that a larger percentage of that series' data has that particular rating or better. For example, $100 \%$ of the TTs have a rating of 3 or better; while only about $30 \%$ of the terms of the lowest-scoring KW document received a score of 3 or better. In two out of the three documents, HS terms fall between TTs and KWs.

\subsection{Coverage}

The graph in Figure 3 shows results for quality, not coverage. In contrast, Figure 4, which shows the total number of terms rated at or below specified rankings, allows us to measure quality and coverage. ( 1 is the highest rating; 5 is the lowest.) This figure shows that the HS method identifies more high quality terms than the TT method does.

\begin{tabular}{|l|cccc|}
\cline { 2 - 5 } \multicolumn{1}{c|}{} & \multicolumn{4}{c|}{$\begin{array}{c}\text { Number of terms ranked } \\
\text { at or better than }\end{array}$} \\
\hline Method & $\mathbf{2}$ & $\mathbf{3}$ & $\mathbf{4}$ & $\mathbf{5}$ \\
\hline KW & 27 & 75 & 124 & 166 \\
HS & 41 & 96 & 132 & 160 \\
IT & 15 & 21 & 21 & 21 \\
\hline
\end{tabular}

Figure 4: Running total of terms identified at or below a specified rank

TT clearly identifies the highest quality terms: $100 \%$ of TTs receive a rating of 2 or better. However, only 8 TTs received a rating of 2 or better (38\% of the total), while 41 HSs re- ceived a rating of 2 or better ( $26 \%$ of the total). This indicates that the TT method misses many high quality terms. $\mathrm{KW}$, the least discriminating method in terms of quality, also provides better coverage than does TT.

This result is consistent with our observation that TT identifies the highest quality terms, but there are very few of them: an average of 7 per 500 words compared to over 50 for HS and KW. Therefore there is a need for additional high quality terms. The list of HSs received a higher average rating than did the list of KWs, as shown in Figure 2. This is consistent with our expectation that phrases containing more content-bearing modifiers would be perceived as more useful index terms than would single word phrases consisting only of heads.

\subsection{Ranking variability}

The difference in the average ratings for the list of KWs and the list of head-sorted SNPs was less than expected. The small difference in average ratings for the HS list and the $\mathrm{KW}$ list can be explained, at least in part, by two factors: 1) Differences among professionals and students in inter-subject agreement and reliability; 2) A discrepancy in the rating of single word terms across term types.

22 students and 7 professionals participated in the study. Figure 5 shows differences in the ratings of professionals and of students.

\begin{tabular}{|l|c|c|}
\hline & Professionals & Students \\
\hline KW & 2.64 & 3.30 \\
\hline HS & 2.3 & 3.03 \\
\hline TT & 1.49 & 2.1 \\
\hline
\end{tabular}

Figure 5: Average ratings, by term type, of professionals and students

When variation in the scores for terms was calculated using standard deviation, the standard deviation for the professionals was 0.78 , while for the students it was 1.02. Because of the relatively low number of professionals, the standard deviation was calculated only over terms that were rated by more than one professional. A review of the students' results showed that they appeared not to be as careful as the professionals. For example, the phrase 'Wall 
Street Journal' was included on the HS list only because it is specified as the document source. However, four of the eight students assigned this term a high rating ( 1 or 2 ); this is puzzling because the document is about asbestos-related disease. The other four students assigned a 4 or 5 to 'Wall Street Journal', as we expected. But the average score for this term was 3 , due to the anomalous ratings. We therefore have more confidence in the reliability of the professional ratings, even though there are relatively few of them.

We examined some of the differences in rating for term types. Single word index terms are rated more highly by professionals when they appear in the context of other single word index terms, but are downrated in the context of phrasal expansions that make the meaning of the one-word term more specific. The KW list and HS list overlap when the SNP consists only of a single word (the head) or only of a head modified by determiners. When the same word appears in both lists in identical form, the token in the KW list tends to receive a better rating than the token does when it appears in the HS list, where it is often followed by expansions of the head. For example, the word exposure received an average rating of 2.2 when it appeared on the KW list, but a rating of only 2.75 on the HS list. However, the more specific phrase racial quotas, which immediately followed quota on the HS list received a rating of 1.

To better understand these differences, we selected 40 multi-word phrases and examined the average score that the phrase received in the TT and HS lists, and compared it to the average ratings that individual words received in the KW list. We found that in about half of the cases ( 21 of 40 ), the phrase as a whole and the individual words in the phrase received similar scores, as in Example 1 in Figure 6. In just over one-fourth of the cases (12 of 40 ), the phrase scored well, but scores from the individual words were rated from good to poor, as in Example 2. In about one-eighth of the cases ( 6 of 40), the phrase scored well, but the individual words scored poorly, as in Example 3. Finally, in only one case, shown in Example 4 of Figure 6, the phrase scored poorly but the individual words scored well.

\begin{tabular}{|c|c|c|c|}
\hline & Phrase & Word 1 & Word 2 \\
\hline 1 & $\begin{array}{l}\text { Supreme Court } \\
(1.5)\end{array}$ & $\begin{array}{r}\text { Supreme } \\
\text { (1) }\end{array}$ & $\begin{array}{l}\text { Court } \\
(1.25)\end{array}$ \\
\hline 2 & $\begin{array}{l}\text { reverse discrimi- } \\
\text { nation } \\
\text { (1) }\end{array}$ & $\begin{array}{l}\text { reverse } \\
\quad(3.25)\end{array}$ & $\begin{array}{c}\text { discrimi- } \\
\text { nation } \\
(3.25)\end{array}$ \\
\hline 3 & $\begin{array}{l}\text { lymph system } \\
\text { (1) }\end{array}$ & $\begin{array}{l}\text { lymph } \\
\text { (1) }\end{array}$ & $\begin{array}{l}\text { system } \\
\text { (5) }\end{array}$ \\
\hline 4 & $\begin{array}{l}\text { employment } \\
\text { decisions } \\
(2.75)\end{array}$ & $\begin{array}{l}\text { employ- } \\
\text { ment } \\
(1.25)\end{array}$ & $\begin{array}{l}\text { decisions } \\
(1.25)\end{array}$ \\
\hline
\end{tabular}

Figure 6: Comparison of scores of phrases and single words

This shows that single words in isolation are judged differently than the same word when presented in the context of a larger phrase. These results have important implications in the design of indexing tools.

\section{Conclusion}

Our results show that the head sorting technique outperforms two other indexing methods, technical terms and keywords, as measured by balance of quality and coverage. We have performed a qualitative evaluation of three techniques for identifying significant terms in a document, driven by an indexing task. Such an application can be used to create a profile or thumbnail of a document by presenting to users a set of terms which can be considered to be a representation of the content of the document. We have used human judges to evaluate the effectiveness of each method. This research is a contribution to the overall evaluation of computational linguistic tools in terms of their usefulness for human-oriented computational applications.

\section{References}

Boguraev, Branimir and Kennedy, Christopher (1998) "Applications of term identification terminology: domain description and content characterisation", Natural Language Engineering 1(1):1-28.

Church, Kenneth Ward (1988) "A stochastic parts program and noun phrase parser for unrestricted text", in Proceedings of the Second 
Conference on Applied Natural Language

Processing, pp.136-143.

Evans, David A. and Chengxiang Zhai (1996)

"Noun-phrase analysis in unrestricted text for information retrieval", Proceedings of the 34th Annual Meeting of the Association for Computational Linguistics, pp.17-24. 24-27 June 1996, University of California, Santa Cruz, California, Morgan Kaufmann Publishers.

Evans, David K. (1998) LinkIT Documentation, Columbia University Department of Computer Science Report.

Evans, David K., Klavans, Judith, and Wacholder, Nina (2000) "Document processing with LinkIT", RIAO Conference, Paris, France, to appear.

Hatzivassiloglou, Vasileios, Judith L. Klavans and Eleazar Eskin (1999) "Detecting text similarity over short passages: exploring linguistic feature combinations via machine learning", Proceedings of the EMNLPNLC99 Joint SIGDAT Conference on Empirical Methods in NLP and Very Large Corpora, June 21-22, 1999, University of Maryland, College Park, MD.

Hodges, Julia, Shiyun Yie, Ray Reighart and Lois Boggess (1996) "An automated system that assists in the generation of document indexes", Natural Language Engineering 2(2): 137-160.

Jackendoff, Ray (1977) X-bar Syntax: A Study of Phrase Structure, MIT Press, Cambridge, MA.
Justeson, John S. and Slava M. Katz (1995)

"Technical terminology: some linguistic properties and an algorithm for identification in text", Natural Language Engineering 1(1):9-27.

Luhn, Hans P. (1958) "The automatic creation of literature abstracts", IBM Journal, 159-165.

Mosteller, Frederick and David L. Wallace (1963) "Inference in an authorship problem", Journal of the American Statistical Association 58(302):275-309. Available at http://www.jstor.org/.

Paice, Chris D. (1990) "Constructing literature abstracts by computer: techniques and prospects". Information Processing \& Management 26(1):171-186.

Salton, Gerald (1989) Automatic Text Processing: The Transformation, Analysis and Retrieval of Information by Computer. AddisonWesley, Reading, MA.

Smadja, Frank (1993) "Retrieving collocations from text", Computational Linguistics 19(1):143-177.

Strzalkowski, Thomas (1997) "Building effective queries in natural language information retrieval", Proceedings of the ANLP, ACL, Washington, DC., pp.299-306.

Wacholder, Nina (1998) "Simplex NPS sorted by head: a method for identifying significant topics within a document", Proceedings of the Workshop on the Computational Treatment of Nominals, pp.70-79. COLING-ACL '98, Montreal, Canada, August 16, 1998.

Figure 3: Cumulative ranking of terms, by method

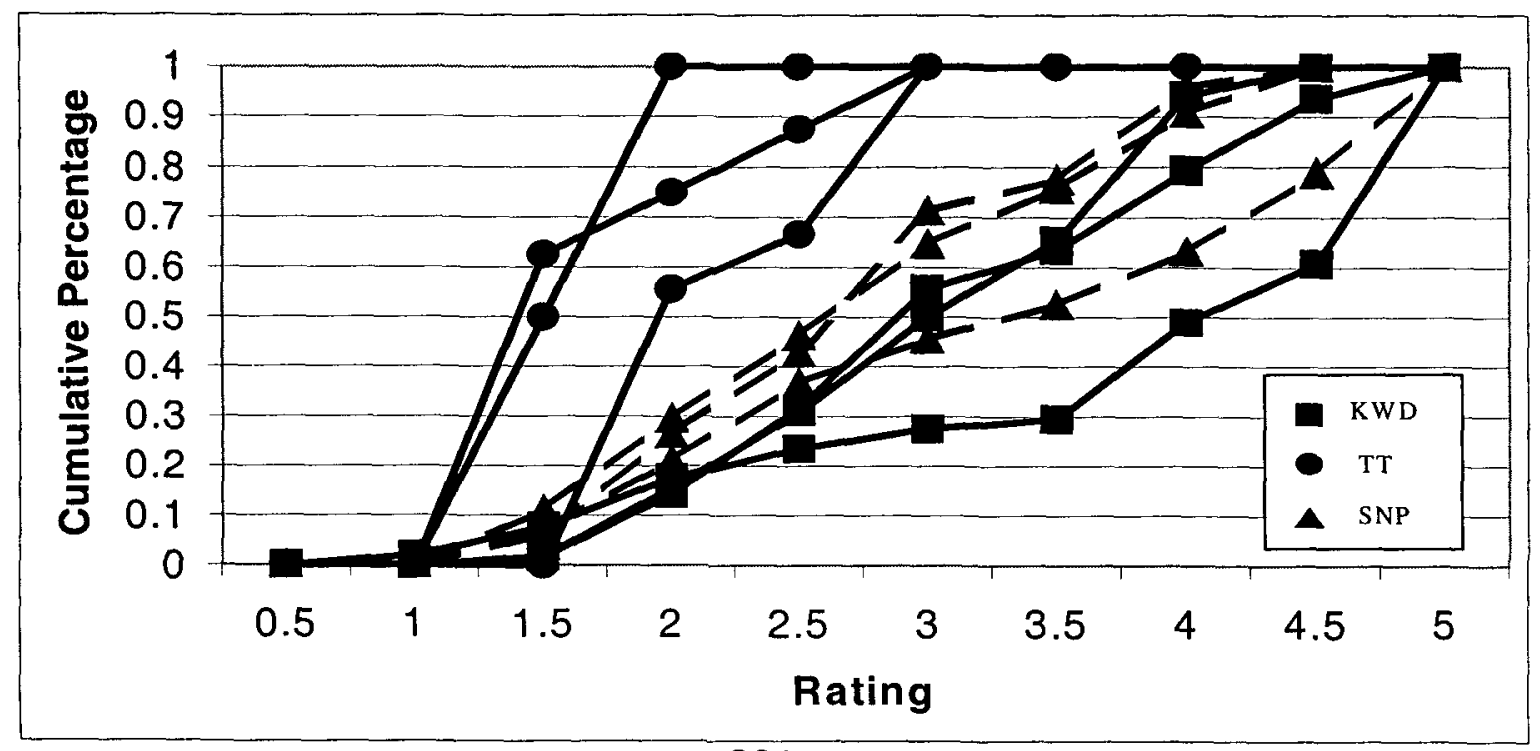


Appendix A: Terms identified in WSJ900405-0109

HSs

amendments

Hatch amendment

other amendments

attempts

bias

job bias

intentional bias

bill

committee

Senate labor Committee

court

Supreme Court

co-workers

decisions

Supreme Court decisions

employment decisions

Democrats

discrimination

reverse discrimination

employees

women employees

employers

groups

civil-rights groups

conservative policy

groups

Orrin Hatch

health

discriminatory impact

Job-Bias Measure

basic employment antidiscrimination law

1866 civil-rights law

lawsuits

lawmakers

legislation

comprehensive legislation

more modest measure

minority/minorities

panel

plans

court-approved affirmative action plans

discriminatory seniority plans

practices

employment practices

quotas

racial quotas

right/rights

equal rights

year

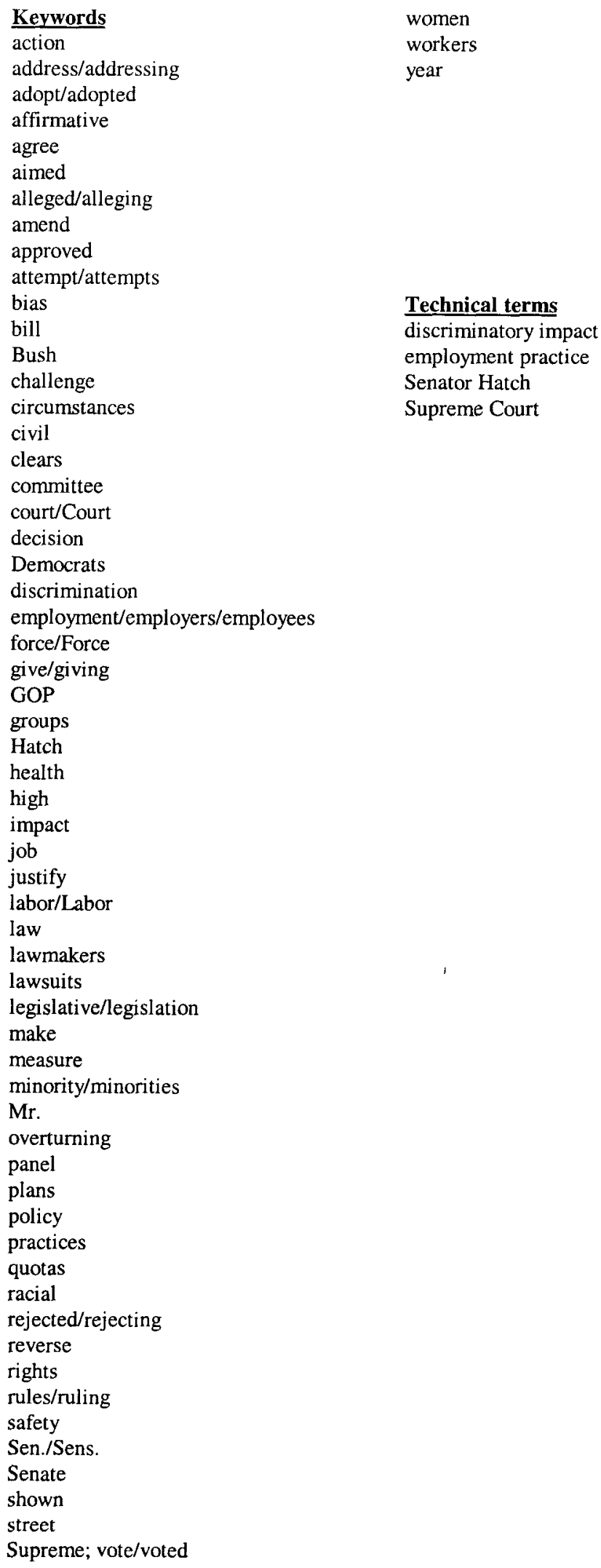

Keywords

action

address/addressing

adopt/adopted

affirmative

agree

aimed

alleged/alleging

amend

approved

attempt/attempts

bias

bill

Bush

challenge

circumstances

civil

clears

committee

court/Court

decision

Democrats

discrimination

employment/employers/employees

force/Force

give/giving

GOP

groups

Hatch

health

high

impact

job

justify

labor/Labor

law

lawmakers

lawsuits

legislative/legislation

make

measure

minority/minorities

Mr.

overturning

panel

plans

policy

practices

quotas

racial

rejected/rejecting

reverse

rights

rules/ruling

safety

Sen./Sens.

Senate

shown

street

Supreme; vote/voted

women

workers

year

Technical terms

discriminatory impact employment practice

Senator Hatch

Supreme Court 